\title{
RF Heating in Cavity-Bellows of CW SRF Cryomodule
}

\author{
A. Saini ${ }^{\dagger}$, A. Lunin, N. Solyak, A. Sukhanov, V. Yakovlev, \\ Fermi National Accelerator Laboratory, Batavia, IL, USA,
}

\begin{abstract}
In a cryomodule, bellows are commonly used to connect two adjacent superconducting cavities. It provides cavities a mechanical flexibility facilitating for thermal contraction or expansion during temperature transitions. While heat from superconducting cavities are removed from helium flowing through their jackets, non-superconducting bellows are cool-down through an indirect process of conduction cooling. An excessive heat deposition to the bellows could results in unsustainable rise in temperature that could lead to a thermal runaway. Because of the bellows proximity to the cavities, resulting thermal runaway could disrupt performance of the cavities and therefore, overall operation. Consequently, a careful evaluation of RF heating in bellows needs to be performed especially for a Continuous Wave (CW) and high intensity beam operation where beam induced, and operating fields heating could be substantial. For this reason, the paper presents a comprehensive analysis of RF heating mechanisms in bellows of $1.3 \mathrm{GHz}$ cryomodule of the Linac Coherent Light Source-II (LCLS-II) linear accelerator (linac). The paper details RF heating estimations for two type of bellows i.e. stainlesssteel and copper bellows and describes simplified analytical approach developed to evaluate temperature distribution across the bellows. Since copper does not exhibit desired material property for the bellows at cryogenic temperature, inner copper coated stainless-steel bellows is an ideal practical alternative. The paper also converses implications of coating on conduction resistance of the multilayer system that enables to determine the coating thickness.
\end{abstract}

Index Terms - Cryomodule, SRF cavity, bellows, RF heating, higher order modes, wake fields, linac, $\mathrm{CW}$, copper coating

\section{INTRODUCTION}

The particle accelerator-based next generation light sources require a high energy electron beam with high repetition rate to produce brighter $\mathrm{x}$-rays that eventually be a tool to enhance our understanding about rare biological and chemical processes. Superconducting Radio Frequency (SRF) technology is foremost choice for such applications involving accelerators operating in long-pulse or continuous-wave (CW) RF regimes. Newly proposed or under construction light-source facilities such as Linac Coherent Light Source-II (LCLS-II) [1-2], European X-rays Free Electron Laser (XFEL) [3], Shanghai Coherent Light Facility (SCLF) [4] are based on the SRF linear accelerator. It is evident that the major infra-structure investment and operational costs of the SRF accelerator are primarily outlined by its cryogenic requirements. Consequently, it is critical not only to evaluate cryogenic heat loads originating

†asaini@fnal.gov, Phone Number +6308405293,

Fermi National Accelerator Laboratory, Batavia IL, 60510 from various sources with a great care but also to constraint them at minimum especially in the cavity operating environment of $2 \mathrm{~K}$ where cryogenic efficiency of removing a Watt of dissipated power is least.

The SRF cavities are building blocks of the accelerator. These cavities are accommodated in the cryomodules. Two successive cavities in a cryomodule are connected through the bellows that provides a mechanical flexibility allowing for length contraction (expansion) during a cool-down (warm-up) of the cavities. While RF heat from surface of the cavity is removed by helium flowing through the helium jacket, heat from beam pipe and bellows are removed through a relatively slow process of conduction cooling. Thus, an excessive heating of the bellows could cause an unsustainable rise in its temperature. Because of close proximity of niobium beam pipe and the SRF cavity to the bellows, an abrupt increase in temperature could produce conduction heating that might lead to increase in BCS surface resistance, $R_{B C S}$, of the niobium [5] that scales with temperature as follows

$$
R_{B C S}(T) \propto \frac{1}{T} f_{0}^{2} e^{-1.76 \frac{T_{c}}{T}}
$$

where $f_{0}$ is operating frequency of the cavity in this case, $T_{c}, T$ are critical temperature and surface temperature respectively. A high surface resistance implies more power dissipation and therefore, more heating. This in turn, not only enhances the cryogenic load at $2 \mathrm{~K}$ environment but also limits $\mathrm{RF}$ performance of the cavities. The accumulated heat in the beam pipe could eventually lead to quenching of the beam pipe and then, the cavity. This is why a careful evaluation of RF heating in a non-superconducting bellows in vicinity of the superconducting elements needs to be performed. It becomes mandate especially for $\mathrm{CW}$ and high intensity beam operation where heating resulting from wake fields [6] and operating fields could be substantial and could disrupt overall operation if have not been accounted properly in the design. For this reason, the paper thoroughly addresses most imperative dynamic heating mechanisms in the bellows induced by the beam and operating fields. The heating is then computed for the $1.3 \mathrm{GHz}, \mathrm{CW}$, SRF cryomodule of the LCLS-II linac considering two types of bellows i.e. stainless-steel bellows and copper bellows. Furthermore, the paper details resulting temperature distribution across the bellows and discusses implication of inner copper coating over the stainless-steel bellows on conduction resistance of the layered system.

The paper is organized in six sections. In Section-II, a brief overview of the LCLS-II operational parameters and $1.3 \mathrm{GHz}$ cryomodule is provided. Section-III systematically details bellows heating resulting from operating mode fields of the cavity, wake-fields and resonance excitation of higher order modes in the LCLS-II cryomodule. An analytical approach

This manuscript has been authored by Fermi Research Alliance, LLC under Contract No. DE-AC02-07CH11359 with the U.S. Department of Energy, Office of Science, Office of High Energy Physics. 
developed to calculate temperature distribution in the bellows is presented in Section-IV while implications of copper coating on stainless-steel bellows are discussed in the Section-V. The paper concludes with a summary in Section-VI.

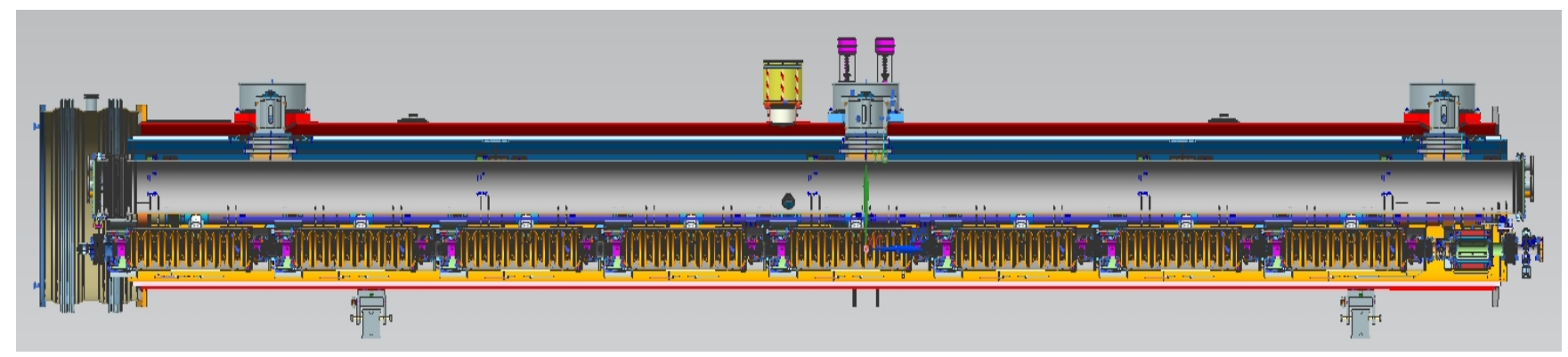

(a)

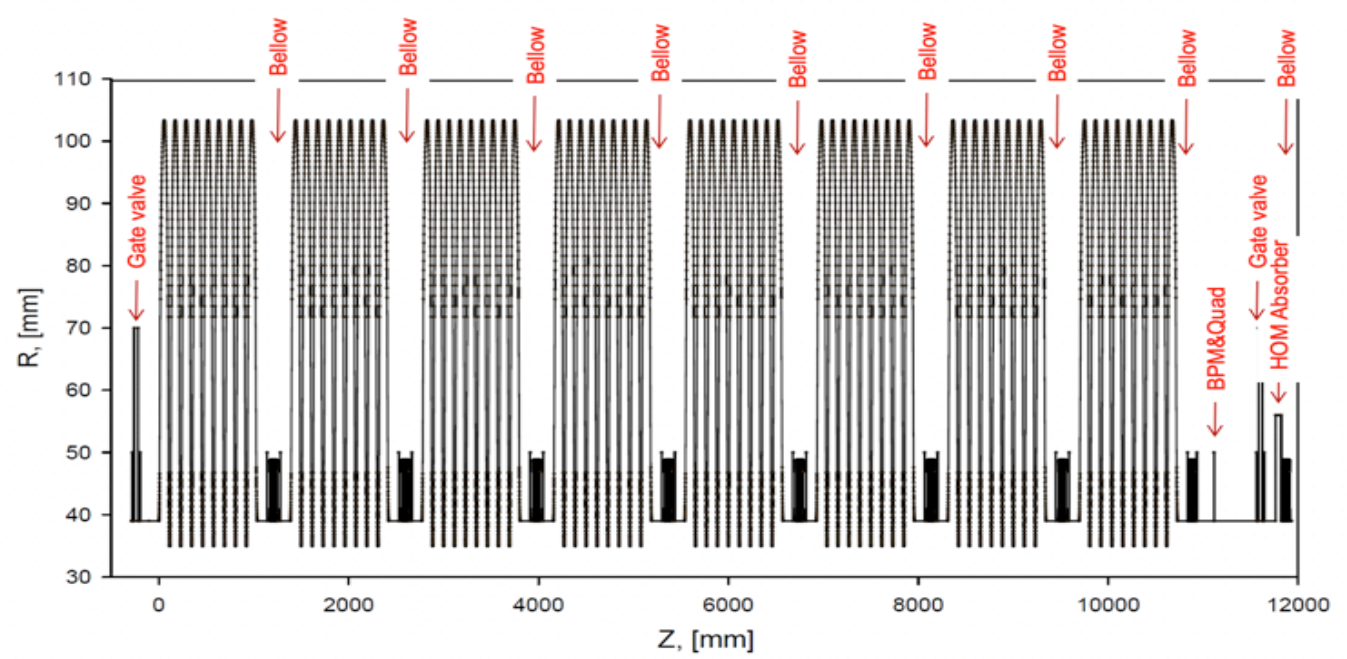

(b)

Figure1: (a) 3D rendering of the $1.3 \mathrm{GHz}$, LCLS-II SRF cryomodule and (b) its detailed schematic showing the cavity-bellows between two adjacent cavities and geometrical variation in vacuum chamber along the cryomodule.

\section{II. $1.3 \mathrm{GHZ}$ LCLS-II CRYOMODULE}

The LCLS-II is a Free Electron Laser (FEL) facility under construction at SLAC national accelerator laboratory in USA. The facility is primarily based on a $4 \mathrm{GeV}$, SRF electron linear accelerator that would operate in a $\mathrm{CW}$ regime. To obtain the designed beam acceleration, the linac uses thirty-five $1.3 \mathrm{GHz}$ cryomodules. Each cryomodule consists of eight 9-cells, 1.3 $\mathrm{GHz}$ elliptical shaped cavities developed for International Linear Collider (ILC) and TESLA projects [7-9]. In addition, the cryomodule also includes a magnet package comprising a quadrupole magnet, horizontal and vertical steering correctors and, the beam position monitor at its downstream end. Figure 1 shows schematic of the LCLS-II, $1.3 \mathrm{GHz}$ cryomodule highlighting some of its crucial elements and cross-sectional variations in vacuum chamber geometry along the linac. It will be discussed in subsequent section that these geometrical variations are primary source of scattered wake fields as the beam traverses through them along the cryomodule. To minimize the wake fields heating in $2 \mathrm{~K}$ environment, a HOM absorber is installed at $70 \mathrm{~K}$ between two cryomodules in the beamline.

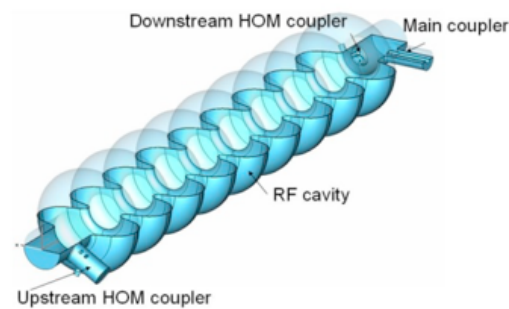

Figure 2: Schematic of a $1.3 \mathrm{GHz}$ cavity with power and HOMs couplers.

The accelerating cavities in the LCLS-II cryomodule operate at $2 \mathrm{~K}$ with nominal accelerating gradient of $16 \mathrm{MV} / \mathrm{m}$. As shown in Figure 2, besides a main power coupler, each cavity is equipped with two Higher Order Modes (HOMs) couplers that facilitate extraction of HOMs fields from $2 \mathrm{~K}$ environment and therefore, limit HOMs induced heating in the cavity. Figure 3 shows the cavity-bellows assembly that connects two adjacent cavities in the LCLS-II cryomodule. It includes eight convolutions and having a physical length, $L_{B}$, of $100 \mathrm{~mm}$. Because of convolution, effective length of the bellows, $L_{B E}$, is larger than its physical length. It can ne estimated using following equation:

$$
L_{B E}=L_{B}+\left(L_{C} / L_{P}-1\right) 2 L_{P} N_{c}
$$


where, $N_{c}$ is number of convolutions of the bellows. As one can notice from Figure 3, $L_{P}$ is half period lateral length of the convolution expressed as:

$$
L_{P}=R_{s m}+R_{L}+t
$$

where, $R_{s m}, R_{L}$ are inner radii of the convolutions and $t$ is the bellows thickness. $L_{C}$ is convoluted length of the half period which is given as follows:

$$
L_{C}=\frac{\pi}{2}\left(R_{s m}+R_{L}+t\right)+(L)
$$

$L$ is length of the straight part of the convolution. Effective length of the cavity-bellows in the LCLS-II cryomodule was found to be $0.236 \mathrm{~m}$. It should also be noted that there is one more bellows at the downstream of the cryomodule (Figure 1). It facilitates connection between two successive cryomodules and has a different design from the cavity-bellows. However, we deal only with the cavity-bellows and therefore, the bellows is always referred to the cavity-bellows everywhere in this article.

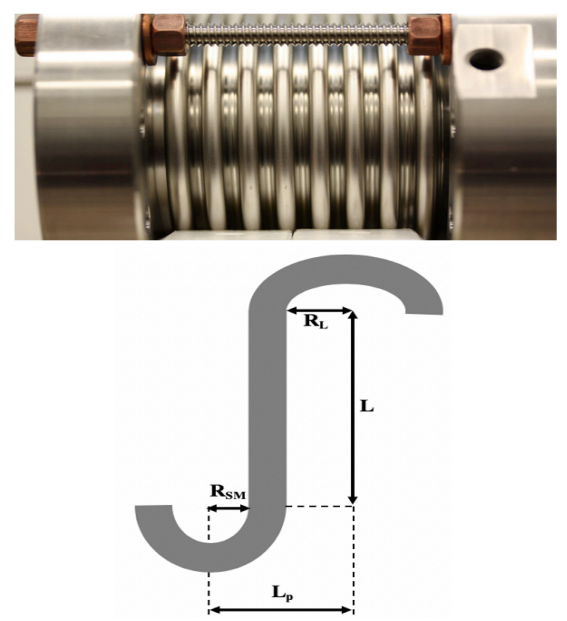

Figure 3: Picture of (top) the cavity-bellows in $1.3 \mathrm{GHz}$ cryomodule and (bottom) a pictorial representation of half period of the convolution geometry.

Most relevant beam and machine parameters of the LCLS-II linac often applied to calculations in this article are listed in Table 1.

TABle 1: Most Relevant Beam Operational Parameters of the LCLS-II SRF LINAC.

\begin{tabular}{cccc}
\hline \hline Parameters & Symbol & Magnitude & Units \\
\hline Bunch Charge & $Q_{\text {bunch }}$ & 300 & $\mathrm{pC}$ \\
Beam Repetition Rate & $f_{\text {rep }}$ & 1 & $\mathrm{MHz}$ \\
RMS Bunch Length & $\sigma_{z}$ & 25 & $\mu \mathrm{m}$ \\
Beam Current & $I$ & 300 & $\mathrm{~A}$ \\
Beam Energy & $E$ & 4 & $\mathrm{GeV}$ \\
Accelerating Gradient in Cavity & $E_{a c c}$ & 16 & $\mathrm{MV} / \mathrm{m}$ \\
Operating Temperature & $T_{o p}$ & 2 & $\mathrm{~K}$ \\
Cavity Operating Frequency & $f$ & 1.3 & $\mathrm{GHz}$ \\
Cavity Beam Pipe Radius & $b$ & 39 & $\mathrm{~mm}$ \\
Bellows physical length & $L_{B}$ & 137 & $\mathrm{~mm}$ \\
Bellows Effective Length & $L_{B E}$ & 236 & $\mathrm{~mm}$ \\
Bellows Thickness & $t$ & 0.2 & $\mathrm{~mm}$ \\
\hline \hline
\end{tabular}

\section{RF HEATING IN CAVITY BELLOWS}

The most potent sources of the bellows heating in a cryomodule are leakage of operating mode fields through the beam holes in the cavity, propagating wake-fields induced by an ultra-relativistic high intensity beam traversing through geometrical variations along the cryomodule and, trapped modes resonance heating. These sources are discussed and analysed in detail in subsequent part of the section to compute the bellow heating in the $1.3 \mathrm{GHz}$, LCLS-II cryomodule.

\section{A. RF Heating due to Operating Mode Field}

The beampipe holes around the axis at both ends of the cavity add a perturbation to ideal modes of the resonator with closed metallic ends. The perturbation is usually small if the modes frequencies are smaller than the cut-off frequency of the beampipe. Still, a small fraction of the operating fields of the accelerator cavity leaks into the beam pipe that result in the RF heating at the bellows.

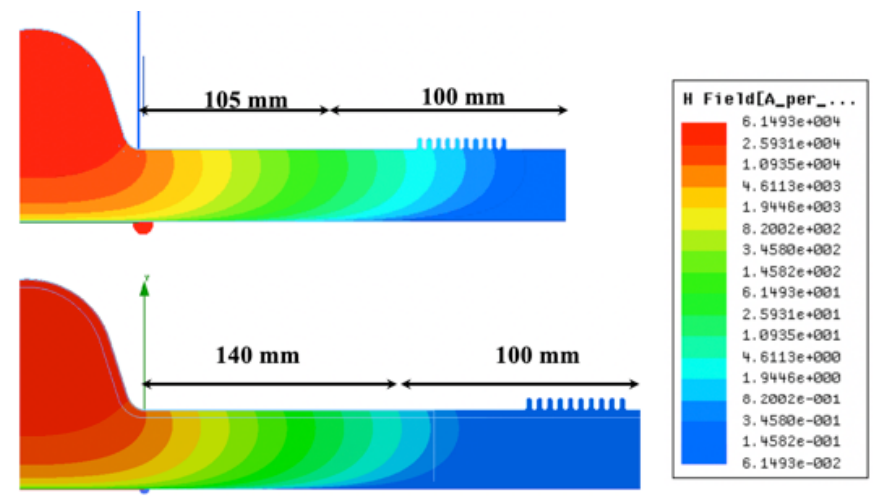

Figure 4: Operating mode magnetic field leaking through the end cell of $1.3 \mathrm{GHz}$ cavity (top) at upstream and (bottom) downstream of the bellows in LCLS-II cryomodule. Note that the fields were normalized to stored energy of $1 \mathrm{~J}$ that were later scaled for nominal accelerating gradient of $16 \mathrm{MV} / \mathrm{m}$.

The operating mode field leakage to the beam pipe from the 1.3 GHz LCLS-II accelerating cavity was computed numerically using the CST field solver [10]. Figure 4 shows pattern of the magnetic fields leaking through the cavity endcells to the bellows at both ends. Note that, cavities have unequal separations from the bellows. The downstream cavity is positioned farther and therefore, only a small fraction of its fields reaches to the bellows. Resulting RF heating $\left(P_{0}\right)$ in the bellows is then computed using following equation:

$$
P_{0}=\frac{1}{2} R_{s} \int H^{2} d s
$$

where $H$ is the magnetic field distribution across the bellows surface, $s$, resulting from the operating mode field decay and $R_{S}$ is the surface resistance of the bellows.

While evaluating RF heating in bellows for the LCLS-II cryomodule, two cases were considered in which the bellows surfaces were assumed to be either stainless steel or copper. The microwave surface resistance is obtained from the real part of the surface impedance which is expressed for stainless steel as shown below 


$$
Z_{s S}(\omega)=(1+i) \sqrt{\frac{\omega \mu_{0}}{2 \sigma}}
$$

where $Z_{s s}$ is surface impedance at angular wave frequency $\omega$, $\mu_{0}$ is magnetic permeability in free space and $\sigma$ is electrical conductivity. Using RF frequency of $1.3 \mathrm{GHz}, \sigma=$ $10^{6}\left(1 / \Omega m^{2}\right)$, accelerating field of $16 \mathrm{MV} / \mathrm{m}$, RF heating in the stainless-steel bellows was found to be $0.1 \mathrm{~W}$.

It has been discussed elsewhere [11] that Copper with the Residual Resistive Ratio (RRR) value of a hundred exhibits extreme Anomalous Skin Effect (ASE) at frequency above 2 $\mathrm{MHz}$ at cryogenic temperature of $4 \mathrm{~K}$. The surface impedance of copper $\left(Z_{c u}\right)$ in this regime is calculated from following equation:

$$
Z_{c u}(\omega)=A(1-\sqrt{3} i) \omega^{\frac{2}{3}}
$$

where $A$ is material constant and, for the copper it is found to be $3.310^{-10}\left(\Omega s^{\frac{2}{3}}\right)$. It is evident from equations (6) and (7) that the surface resistance of copper is about fifty times lower than stainless steel for given parameters at $1.3 \mathrm{GHz}$. Consequently, RF heating in the copper bellows is lower than the stainless steel below by the same factor and found to be $2 \mathrm{~mW}$. Furthermore, estimations of RF heating in two stainlesssteel flanges in the bellows assembly were performed. It was found that operating mode fields resulted in RF heating of 75 $m W$ per flange.

\section{B. Wake-Fields Heating}

An ultra-relativistic charged-particle beam moving on the axis of a round beam pipe induces image charges on its surface. These image charges navigate alongside of the beam and create the image current. Because of a finite conductivity of the surface and cross-sectional variation of the geometrical boundaries along the beam path, the image current flow lags behind the beam. This in turn, results in trailing electromagnetic fields behind the beam that are called wake-fields.

The beam encounters several geometrical variations as it traverses through the cryomodule. These variations (as shown in Fig.1) appear in form of cavities, bellows, beam pipe in between, gate valves etc. Consequently, the beam generates scattered wake-fields (also called radiative wake-fields) as its passes through the cryomodule. These fields eventually dissipate and generate heating at various surfaces along the beamline. Total power instituted through the wake fields, $P_{\text {wake }}$, is given as [12]:

$$
P_{\text {wake }}=Q_{\text {bunch }}^{2} \kappa \frac{T_{\text {pulse }}}{T_{b}} f_{\text {rep }}
$$

where $Q_{\text {bunch }}$ is the bunch charge, $\kappa$ is the loss factor that determines energy lost by a bunch of unit charge in a single passage through the structure, $T_{p u l s e}$ and $T_{b}$ are the beam pulse length and bunch spacing respectively and, $f_{\text {rep }}$ is the bunch repetition rate. A detailed analysis presented elsewhere [12-13] shows that the loss factor per cryomodule of the LCLS-II has been estimated to $154 \mathrm{~V} / \mathrm{pC}$ for a Gaussian bunch of RMS length of $25 \mu \mathrm{m}$. It results in a steady state total wake power of $13.8 \mathrm{~W}$ per cryomodule for the bunch charge of $300 \mathrm{pC}$ and repetition rate of $1 \mathrm{MHz}$. Note that, the LCLS-II linac operates in $\mathrm{CW}$ regime where a single bunch traverse through the linac with the repetition rate of $1 \mathrm{MHz}\left(T_{p u l s e}=T_{b}\right)$.

In frequency domain, frequency range of the wake-fields can be approximated [14] as $f \approx \frac{c}{\sigma_{z}}$; where $c$ is velocity of light. In the LCLS-II linac, the RMS bunch length can be as short as 25 $\mu \mathrm{m}$ and therefore, the wake field frequency spectrum extends up to Tera-Hz. Thus, it is more appropriate to express the total wake power as following:

$$
P_{\text {wake }}=\int_{0}^{\omega_{c}} \frac{d P}{d \omega} d \omega+\int_{\omega_{c}}^{\omega_{m}} \frac{d P}{d \omega} d \omega
$$

where $\omega_{c}, \omega_{m}$ are angular cut-off frequency of the cavity beam pipe and the highest frequency of the spectrum respectively. The first term in equation (9) represents the wake power instituted in modes below cut-off frequency $\left(\omega_{c}\right)$. This power is mainly localized in the cavity and, most of it can be effectively extracted from the operating cryogenic environment using HOMs couplers. Thus, it has relatively lower contribution in the overall RF heating. The second term in equation (9) has leading role in the heating. It corresponds to modes which have frequencies above cut-off frequency of the beam pipe. These high frequency modes can propagate freely in the linac and make multiple reflections from surfaces of the beamline elements before getting completely absorbed. In this paper we will call them propagating or un-trapped modes. Since frequency spectrum of the propagating modes spread over terahertz range, estimation of their heating distribution in a cryomodule using standard numerical methods is unfeasible. The S-matrix [15] and diffusion-type model [16] approaches have been developed to address this issue. In this article we use the diffusion model to evaluate heating in bellows resulting from high frequency propagating modes.

\section{1) Diffusion Model}

The diffusion model is based on assumption that the propagating modes can be treated as a photon gas distributed uniformly in the cryomodule. It is also assumed that modes dissipate all their power inside the cryomodule and therefore, no power leakage is anticipated in this model. This assumption is justifiable in presence of the long cryo-string (as it is the case for the LCLS-II linac) where power leaking out from a cryomodule is equal to power coming in from its adjacent cryomodules in a steady state. As stated earlier, the propagating modes make multiple reflections from surfaces of the beamline element and with each reflection they lose a fraction of their power. Consequently, the model considers that the power absorption is proportion to the surface impedance and surface area of elements. Thus, absorption $(I)$ of the wake power in frequency range of $\omega$ to $\omega+d \omega$ by an element of surface area $S$ can be characterized as:

$$
I(\omega) \propto S \frac{d P(\omega)}{d \omega} \operatorname{Re}(Z(\omega)) d \omega
$$

where $\operatorname{Re}(Z(\omega))$ is real part of the element impedance at frequency $\omega$. Differential wake power, $\frac{d P(\omega)}{d \omega}$, is computed using:

$$
\frac{d P(\omega)}{d \omega}=\frac{1}{\pi} Q_{\text {bunch }}^{2} f_{\text {rep }} \operatorname{Re}\left[Z_{w}(\omega)\right] e^{\left(-\frac{\omega \sigma_{z}}{c}\right)^{2}}
$$


where wake impedance, $Z_{w}$ is given by

$$
Z_{w}=\frac{1}{c} \int_{0}^{\infty} W_{\|}^{0}(s) e^{-\left(\frac{i \omega s}{c}\right)} d s
$$

Note that $s$ is position of the particle relative to the bunch center. In order to obtain differential wake power spectrum for the LCLS-II cryomodule, the point charge wake function, $W_{\|}^{0}(s)=344 e^{-\sqrt{\left(\frac{s}{s_{0}}\right)}} V / p C$ with $s_{0}=1.74 \mathrm{~mm}$, of Tesla cryomodule obtained from [17] was used. The resulting power spectrum is shown in Figure 5. Then, power absorption of an $i^{\text {th }}$ element in the cryomodule can be obtain from following expression:

$$
P_{i}=P_{\text {wake }}\left(\frac{I_{i}}{\sum_{j=1}^{n} I_{j}}\right)
$$

where $I$, is absorption amplitude over entire frequency spectrum and subscript $j=1,2,3, \ldots n$, represents elements in the cryomodule. A detailed description of the diffusion model has also been presented in references [18-20].

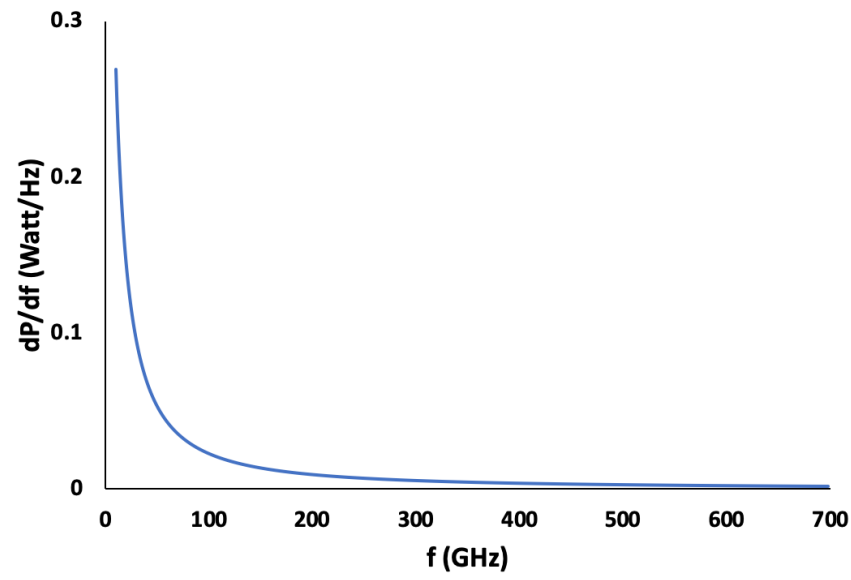

Figure 5: Differential wake-fields power spectrum generated by a $300 p C$ bunch of RMS length of $25 \mu \mathrm{m}$ with a repetition rate of $1 \mathrm{MHz}$ while passing through the $1.3 \mathrm{GHz}$ LCLS-II cryomodule.

To estimate the power absorption in the bellows, surface impedances of stainless steel and copper were computed using equations (6) and (7) over the entire wake-field frequency spectrum. Figure 6 depicts their impedances over the frequency range up to $700 \mathrm{GHz}$. Then, the absorption amplitude of bellows in the LCLS-II cryomodule was computed by integrating equation (10) over the entire frequency spectrum using the knowledge of $\frac{d P(\omega)}{d \omega}$, surface area for radius of $39 \mathrm{~mm}$ and effective length of $236 \mathrm{~mm}$. The absorption amplitude of rest of elements in the cryomodule were computed using same approach and then applied to equation (13) to determine their respective power absorption. It was found that a stainless-steel bellows intercepted about $0.3 \mathrm{~W}$ of total wake-fields power of $13.8 \mathrm{~W}$ while a copper bellows absorbed only $25 \mathrm{~mW}$. RF heating in stainless-steel flanges was found to be $40 \mathrm{~mW}$.

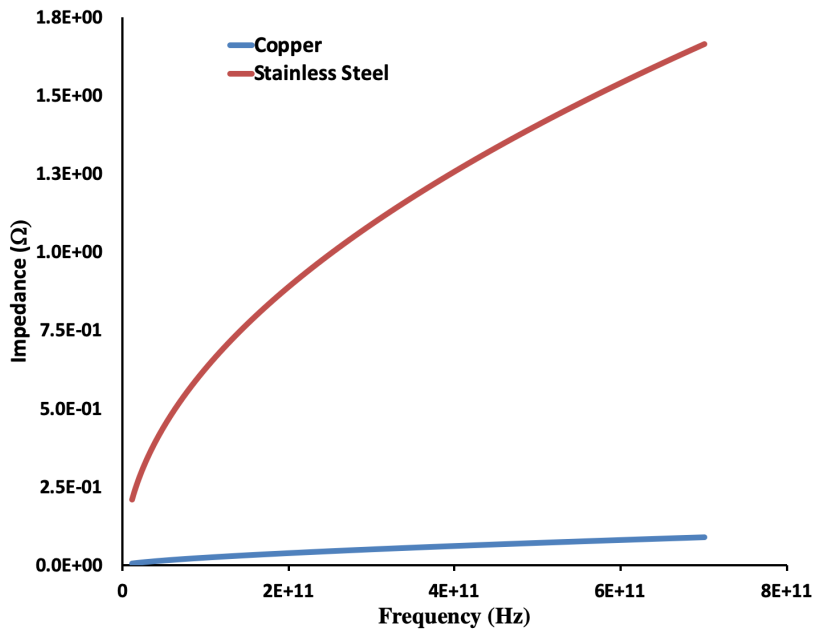

Figure 6: Variation in surface impedance of copper (blue) and stainless steel (brown) with frequency. Note that anomalous skin effect was accounted for the copper at cryogenic temperature around $4 \mathrm{~K}$.

\section{Trapped Higher Order Modes Heating}

There is a periodic arrangement of the cavities in an SRF cryomodule. These cavities are tuned to operating mode frequency, but their higher order modes frequency spectrum can differ due of mechanical variations in their geometries. This might cause reflection of lower frequency propagating modes, usually in vicinity of the beam-pipe cut-off frequency, from the end of adjacent cavities. Reflecting modes could get effectively trapped and form a standing wave in the beam pipe. This in turn, results in localized resonance RF heating in the region.

To investigate the trapped modes in interconnecting beampipe region between two adjacent cavities in the LCLS-II cryomodule, an eigen-mode analysis for higher order modes up to $5 \mathrm{GHz}$, was performed using HFSS field solver [21]. Figure 7 shows electric field distribution of the five trapped modes with highest $R / Q$ and external quality factor $\left(Q_{e x t}\right)$. Note that, only half of the cavities ( 4.5 cells out of nine cells) at both ends of the beam pipe were simulated for this analysis. Table 2 summarises RF parameters of the respective modes.

TABLE 2: RF PARAMETERS OF TRAPPED MODES IN BEAM PIPE BETWEEN ADJACENT CAVITIES IN LCLS-II SRF CRYOMODULE

\begin{tabular}{lllll}
\hline $\begin{array}{l}\text { Modes } \\
\text { Number }\end{array}$ & $\begin{array}{l}\text { Frequency } \\
(\mathrm{GHz})\end{array}$ & $\begin{array}{l}\mathrm{R} / \mathrm{Q} \\
\Omega\end{array}$ & $\mathrm{Q}_{\mathrm{ext}}$ & $\begin{array}{l}\mathrm{P}_{\mathrm{tm}} \\
\mathrm{mW}\end{array}$ \\
\hline 1 & 2.714 & 1.7 & $1.2 \mathrm{E} 04$ & 2 \\
2 & 2.928 & 6 & $1.0 \mathrm{E} 04$ & 5 \\
3 & 2.9683 & 1.5 & $1.7 \mathrm{E} 04$ & 2 \\
4 & 3.047 & 1.7 & $8.7 \mathrm{E} 4$ & 13 \\
5 & 3.141 & 1.1 & $1.0 \mathrm{E} 3$ & 1 \\
\hline \hline
\end{tabular}




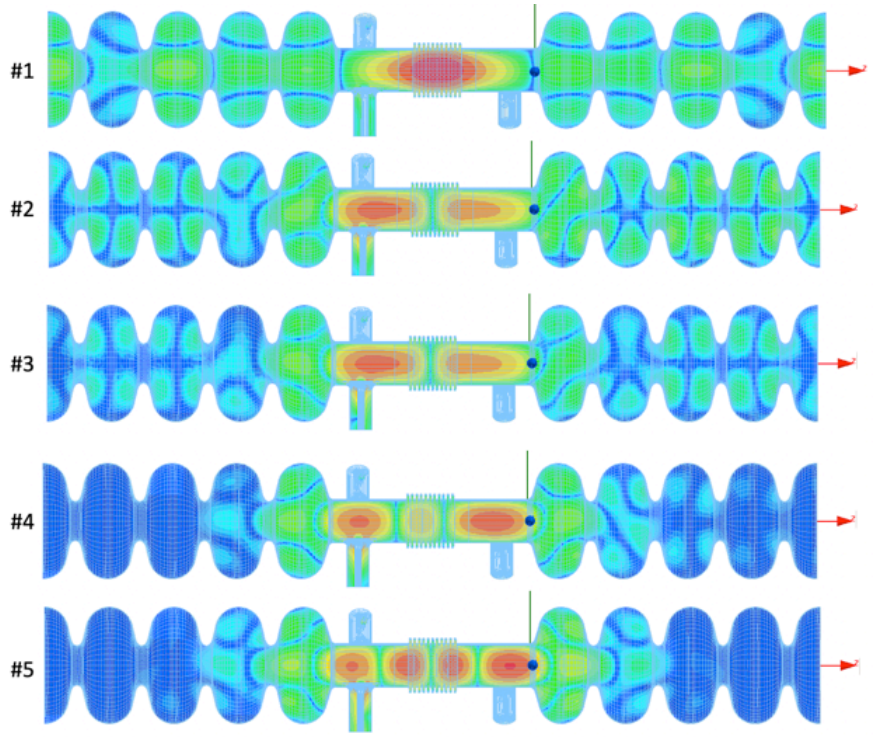

Figure 7: Electric field distributions of trapped modes in the beam pipe region between two adjacent cavities in the LCLS-II cryomodule. RF parameters of respective modes are listed in Table 2. Note that, red colour represents the highest magnitude of the field while blue colour represents the lowest.

Resulting RF heating from a trapped mode, $P_{t m}$, is computed using following equation:

$$
P_{t m}=\left(\frac{R}{Q}\right) Q_{\text {ext }} I_{b}^{2}
$$

where $I_{b}$ is average beam current. It is $300 \mu A$ for the LCLS-II linac (for $Q_{\text {bunch }}$ of $300 \mathrm{pC}$ and $f_{\text {rep }}$ of $1 \mathrm{MHz}$ ). This in turn, results in a maximum heating of $13 \mathrm{~mW}$ for the fourth mode in Table 2. For a mode with $R / Q$ of $10 \Omega$ and $Q_{e x t}$ of $1 E+05$ (highest respective magnitudes in Table 2 were rounded up to the next order), the resonance heating in the worst-case scenario might increase up to $100 \mathrm{~mW}$. Since the trapped modes generate localized RF heating, it is appropriate to assume that magnitude of heating remains same regardless of the bellows types (copper or stainless-steel).

\section{Resistive Wall Heating}

Because of a finite conductivity of the beam-pipe, wakefields generate ohmic losses at its surface which is termed as the resistive wall heating or parasitic heating. The loss factor per unit length, $\kappa_{L}$, in anomalous skin effect regime for shortrange wake-fields approximation is obtained from [11] in form shown below

$$
\kappa_{L}=\frac{3 c Z_{0}}{10 b^{2} \pi^{2}} \int_{0}^{\infty} \frac{e^{-\left(\frac{s^{3} \csc (\pi / 10) \sigma_{Z}}{s_{0}}\right)^{2}}}{s^{2}-\sqrt{3} s+1} d s
$$

where $Z_{0}$ is wave impedance in free space, $b$ is radius of the cylindrical beam pipe and $\mathrm{s}_{0}$ is characteristic length given as

$$
s_{0}=\csc (\pi / 10)(B b)^{3 / 5}
$$

where $B$ is a numerical coefficient determined from material properties. Its value for copper is found to be 3.9E-07 (m $)^{2 / 3}$. For the stainless-steel bellows in a normal skin effect regime, $\kappa_{L}$ is calculated using

$$
\kappa_{L}=\frac{Z_{0} c}{2 \pi b^{2}} e^{-3 \sigma_{Z}^{2} / s_{0}^{2}} ; s_{0}=\left(2 b^{2} / Z_{0} \sigma\right)^{\frac{1}{3}}
$$

The power loss per unit length in copper and stainless bellows are then assessed by inserting respective loss factors in equation (8). Figure 8 shows variation in the resistive wall heating with RMS bunch length for the LCLS-II beam parameters. For the RMS bunch length of $25 \mu \mathrm{m}$, power loss gradient in copper and stainless-steel are about $0.3 \mathrm{~W} / \mathrm{m}$ and $1 \mathrm{~W} / \mathrm{m}$ respectively. This corresponds to total heating of $30 \mathrm{~mW}$ and $100 \mathrm{~mW}$ in respective copper and stainless-steel bellows of length $100 \mathrm{~mm}$.

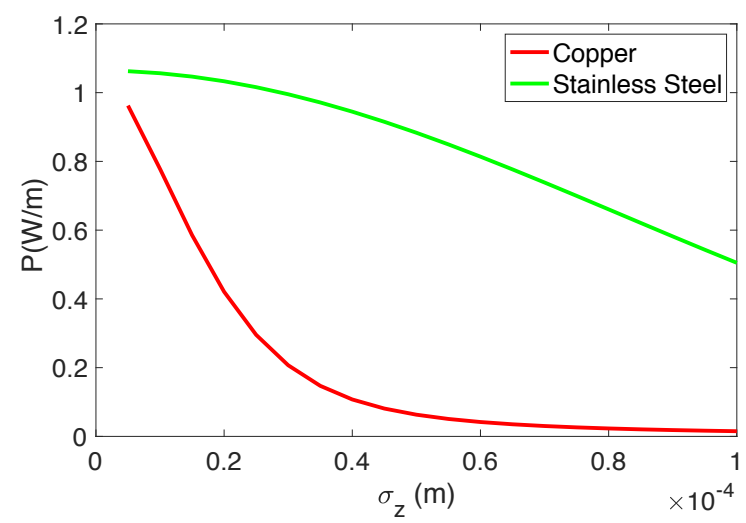

Figure 8: Resistive wall heating per unit length at different RMS bunch lengths for copper (red) in anomalous regime and stainless-steel (green) in normal regime for $Q_{b u n c h}$ of $300 p C$ and $f_{\text {rep }}$ of $1 M H z$.

Table 3 summarizes RF heating in the bellows assembly from various sources in the LCLS-II cryomodule. The heating in copper and stainless-steel bellows were found to be $0.15 \mathrm{~W}$ and $0.6 \mathrm{~W}$ respectively and rest of the heating happened in the flanges made of the stainless-steel. It is evident from here that because of a lower electrical conductivity, stainless-steel surface would cause about four times higher heating in comparison to copper surface in the bellows. Again, note that this heating occurs in $2 \mathrm{~K}$ environment.

\begin{tabular}{ccc}
$\begin{array}{c}\text { TABLE 3: SUMMARY OF RF HEATING IN A CAVITY-BELLOWS } \\
\text { ASSEMBLY IN THE LCLS-II 1.3GHz CRYOMODULE. }\end{array}$ \\
\hline \hline Heating Modes & $\begin{array}{c}\text { Copper } \\
\text { Bellows } \\
(\mathrm{mW})\end{array}$ & $\begin{array}{c}\text { Stainless-Steel } \\
\text { Bellows } \\
\text { (mW) }\end{array}$ \\
\hline $\begin{array}{c}\text { Operating Mode } \\
\text { Fields } \\
\text { Wake Field }\end{array}$ & 152 & 250 \\
Trapped Modes & 65 & 340 \\
Resistive Wall & 30 & 100 \\
Total & 347 & 100 \\
\hline \hline
\end{tabular}

\section{TEMPERATURE DISTRIBUTION IN BELLOWS}

The heat transfer in a high vacuum regime at cryogenic temperature is primary governed by the process of heatconduction. Thus, resulting temperature rise in the bellows 
from the RF heating in a steady state could be obtained analytically by solving the heat-diffusion equation. Assuming the heat transfer from the bellows (a heat source) to the cavity (heat-sink) is mostly in one dimension, 1-D heat diffusion equation is then expressed as below

$$
\frac{\partial}{\partial z}\left(k(T) \frac{\partial T}{\partial z}\right)=-\frac{(\dot{q}(z+\delta z)-\dot{q}(z))}{d z}
$$

Considering a uniform heat deposition, above equation becomes

$$
\frac{\partial}{\partial z}\left(k(T) \frac{\partial T}{\partial z}\right)=-\dot{Q}
$$

where $T, k$ are temperature and the thermal conductivity respectively and, $\dot{Q}$ is rate of heat deposition per unit volume. The solution of above equation with boundary conditions $T(z=0)=T(z=L)=T_{1}$ is expressed as below

$$
\int_{T_{1}}^{T} k(T) d T=-\frac{\dot{Q}}{2}\left(z^{2}-L z\right)
$$

Thermal conductivity of copper with RRR of 30 and stainless steel-304 for a wide range of temperature are shown in Figure 9.
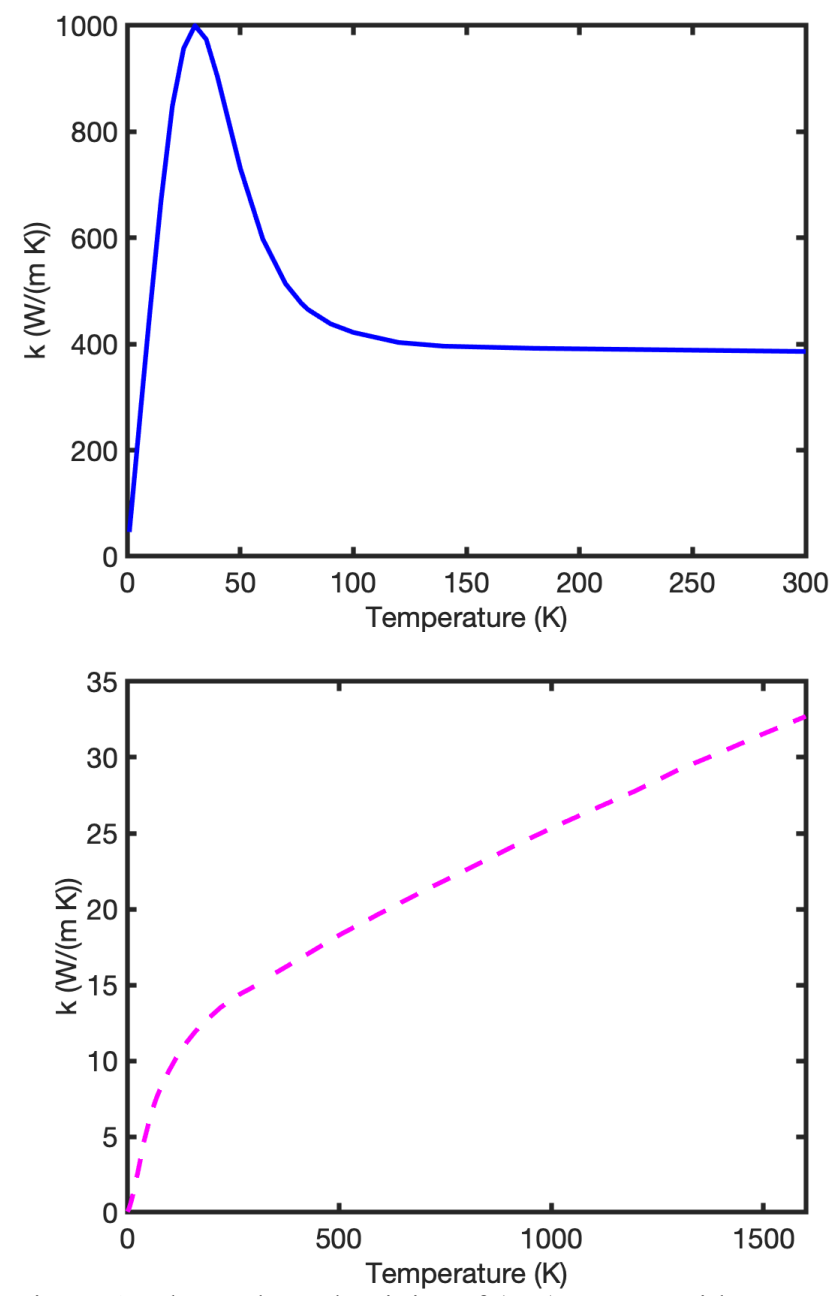

Figure 9: Thermal conductivity of (top) copper with RRR of 30 and, (bottom) stainless steel 304.
The bellows in the LCLS-II cryomodule was modelled as a cylindrical tube of thickness $0.2 \mathrm{~mm}$ and effective length of 236 $\mathrm{mm}$ with a radius of $39 \mathrm{~mm}$. Then, temperature profile of the bellows was obtained from equation 20 . for the heat deposition of $0.15 \mathrm{~W}$ for copper and $0.6 \mathrm{~W}$ for stainless steel. It was assumed that both ends of the bellows were at a fixed temperature of $T_{I}=4 K$. Figure 10 shows temperature evolution in copper and stainless bellows in the LCLS-II cryomodule. It can easily be concluded from Figure 10 that the stainless-steel bellows not only causes more RF heating but also attributes to a substantial rise in temperature due to a lower thermal conductivity at the cryogenic temperature.
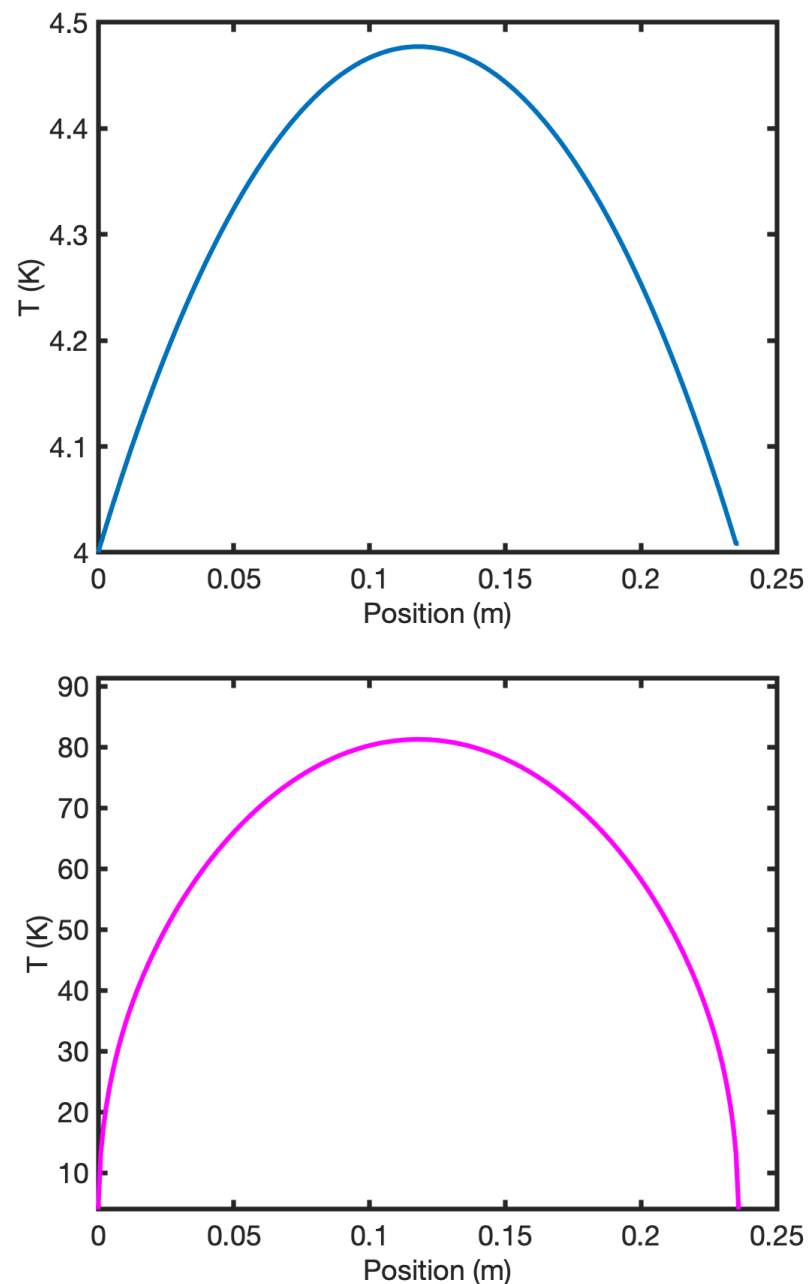

Figure 10: Temperature profile of (top) copper and (bottom) stainless-steel bellows in the LCLS-II SRF cryomodule.

To validate analytical calculation, thermal analysis was performed using ANSYS with a realistic geometry of the bellows. It can be observed from Figure 11 that the maximum temperature in stainless-steel bellows found to be about $72 \mathrm{~K}$ for $0.6 \mathrm{~W}$ of heating. It reasonably agrees with a simplified but less time-consuming analytical model that predicts a maximum temperature of about $80 \mathrm{~K}$. 


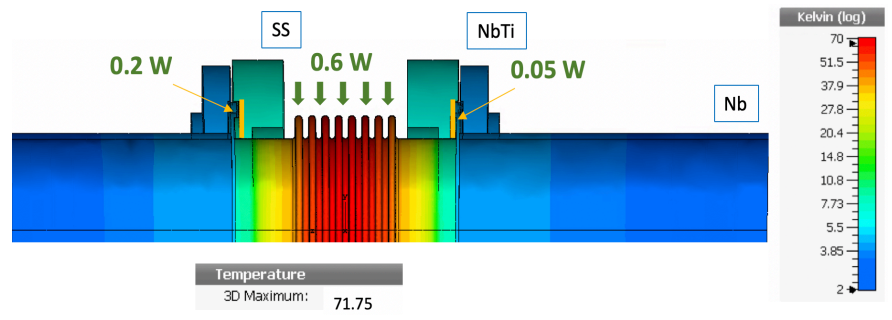

Figure 11: Temperature distribution in stainless-steel bellows for RF heating of $0.6 \mathrm{~W}$.

\section{COPPER COATING}

It is apparent from RF heating and thermal analyses that a copper bellows is more preferable choice over the stainlesssteel bellows. However, copper does not possess optimal material properties (such as tensile strength, yield strength) required for the bellows at cryogenic temperature. Consequently, copper coated stainless-steel bellows is a better practical alternative for the cryomodule. While the copper coated surface provides a higher electrical and thermal conductivities, stainless-steel main frame offers a mechanical stability to the bellows. Note that thermal conductivity of the copper is about 600 times higher than the stainless-steel at $4 K$.

\section{A. Estimation of Coating Thickness}

The conduction resistance of a multilayer coated system (as shown in Figure 12), can be obtained using electric circuit network analogy which is expressed as following:

$$
R_{S}=\frac{L}{k_{1} A_{1}+k_{2} A_{2}}
$$

where $k_{l}$ and $A_{l}$ are thermal conductivity and surface area of cross-section of base material respectively while $k_{2}$ and $A_{2}$ are thermal conductivity and surface area of cross section of coated material respectively. It is apparent from here that a high thermal conductivity implies a lower conduction resistance and therefore a swift heat dissipation. The temperature growth for a given rate of heat deposition $P_{\text {heat }}$ is then evaluated from expression, $\Delta T=P_{\text {heat }} R_{s}$.

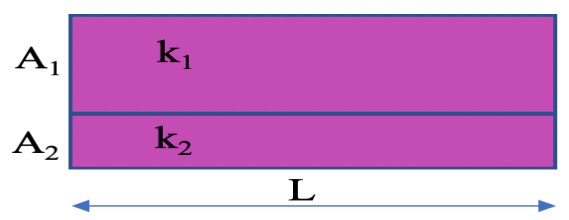

Figure 12: Pictorial representation of a multilayer system.

The maximum temperature rise in a copper coated stainlesssteel bellows for a uniform thermal conductivity is computed using following expression

$$
\Delta T_{\max }=\frac{P_{R F}}{8} \frac{L_{B E}}{k_{1} A_{1}+k_{2} A_{2}}
$$

Note that above equation was obtained after replacing the conduction resistance of a single layer system with coated system in the solution of equation (19) computed for a uniform thermal conductivity.

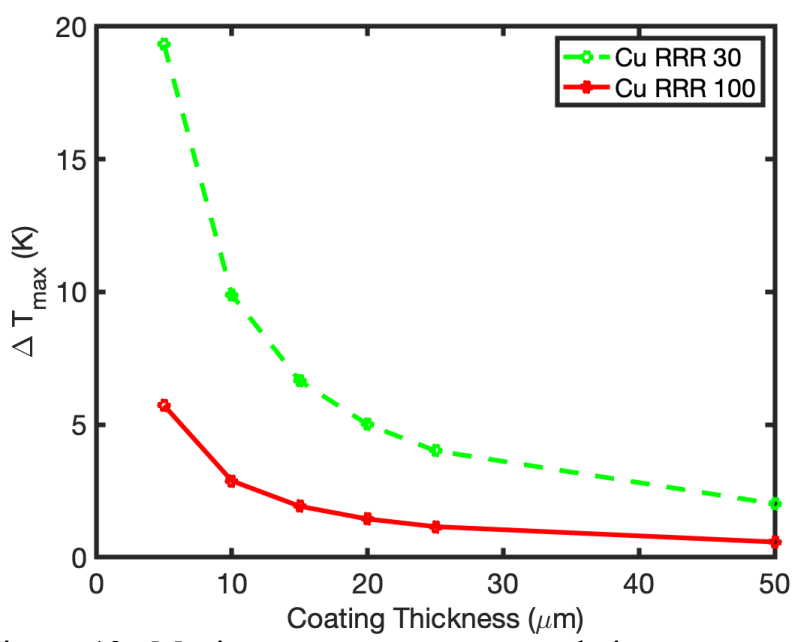

Figure 13: Maximum temperature growth in copper coated stainless-steel bellows in the LCLS-II cryomodule for different coating thicknesses for two types of copper.

Required parameters were inserted in equation (22) to estimate the maximum temperature growth in copper coated stainless-steel bellows in the LCLS-II cryomodule. For total RF heating, $P_{R F}$, equal to $0.15 \mathrm{~W}$ (for copper surface), $k_{1}$ and $k_{2}$ being respective thermal conductivities of stainless-steel and copper which values at $4 \mathrm{~K}$ are 0.22 and $184 \mathrm{~W} /(\mathrm{m}-\mathrm{K})$ respectively, Figure 13 shows maximum temperature growth in the bellows for different copper coating thicknesses. It is apparent from here that a higher coating thickness further lowers the temperature growth. However, coating-thickness can be minimized by using copper with a higher RRR due to increase in its thermal conductivity. Note that copper with RRR of 100 possesses thermal conductivity of about $640 \mathrm{~W} /(\mathrm{m}-\mathrm{K})$ at $4 K$ in comparison to $184 \mathrm{~W} /(\mathrm{m}-\mathrm{K})$ of copper with RRR of 30 [22]. The coating thickness of $15 \mu \mathrm{m}$ has been selected for the bellows in the LCLS-II cryomodule that facilitates to confine maximum temperature growth within $10 \mathrm{~K}$.

\section{SUMMARY}

This paper presented a comprehensive analysis of RF heating in non-superconducting bellows in vicinity of the superconducting cavities. The bellows heating originating from operating fields, wake-fields, trapped modes and resistive wall were computed for $1.3 \mathrm{GHz}, \mathrm{CW}$, LCLS-II cryomodule for two types of the bellows surfaces i.e. copper and stainless-steel. It was found that the stainless-steel bellows resulted in about four times higher heating in comparison to the copper bellows at $2 \mathrm{~K}$ environment. The heating contributes to not only an increase in overall cryogenic loads of the cryomodule but also a temperature growth of the bellows that dissipates its heat through the process of conducting cooling. Study shows that stainless-steel bellows attributes to relatively higher temperature growth of $72 \mathrm{~K}$. Consequently, a copper coating of $15 \mu \mathrm{m}$ over stainless-steel bellows has been selected for the LCLS-II bellows that facilitates not only to minimize RF heating but also to confine temperature growth within $10 \mathrm{~K}$. 


\section{ACKNOWLEDGEMENTS}

The authors would like to express gratitude to the large team of scientists, engineers and technical staffs who were involved in production of $1.3 \mathrm{GHz}$, LCLS-II cryomodule. The authors are also thankful to Karl Bane and Martin Dohlus for useful discussion on wake-fields heating. The author also wishes to acknowledge efforts of Barbara Merrill and Priyanka Saini who proof-read this manuscript.

This manuscript has been authored by Fermi Research Alliance, LLC under Contract No. DE-AC02-07CH11359 with the U.S. Department of Energy, Office of Science, Office of High Energy Physics.

\section{REFERENCES}

[1] J. N. Galayda, "The LCLS-II: A High-Power Upgrade to the LCLS," in proceedings of IPAC2018, Vancouver, Canada, MOYGB2, pp. 18-23.

[2] T. Raubenheimer et al., "LCLS-II Final Design Report," SLAC, CA, USA, Tech. Rep. LCLSII-1.1-DR-0251, 2015.

[3] M.Altarelli et al., “The European X-Ray Free-Electron Laser Technical Design Report,” DESY, Germany, Tech. Rep. 2006-097, ISBN 978-3-93570217-1, July, 2007.

[4] Z. T. Zhao, "SCLF: an 8-GeV CW SCRF LINAC-Based X-RAY FEL Facility in Shanghai," in proceedings of FEL2017, Santa Fe NM, USA, MOP055, pp. 182-184.

[5] H. Padamsee, J. Knobloch and T. Hays, "RF Superconductivity for Accelerators," Jhon Wiley \& Sons Inc., 1998, pp. 1-100.

[6] E. Metral, "RF Heating From Wake Losses In Diagnostics Structures,", in proceedings of IBIC2013, Oxford, UK, THBL1, pp 929-935.

[7] N. Phinney, N. Toge and N. Walker, "ILC Reference Design Report Accelerator Volume 3," 2007.

[8] D. A. Edwards, “TESLA Test Facility Linac-Design Report," DESY, Germany, Rep. 95-01, 1995.

[9] B. Aune et al., "Superconducting Tesla Cavities," Phys.Rev. ST Accel. Beams, vol. 3, no. 09, pp. 092001-25, 09, 2000, DOI: 10.1103/PhysRevSTAB.3.092001

[10] https://www.3ds.com/products-services/simulia/products/cst-studio suite/solvers/

[11] B. Podobedov, "Resistive Wall Wakefields in the extreme anomalous skin effect regime," Phys.Rev. ST Accel. Beams, vol. 12, no. 04, pp. 044401-10, 04, 2009, 10.1103/PhysRevSTAB.12.044401

[12] K. Bane, A. Romanenko and, V. Yakovlev, "Some wakefield effects in the superconducting RF cavities of LCLS-II," SLAC, CA, USA, Rep. SLAC-PUB15932, LCLS-II TN -13-04, March 2014.

[13] K. Bane, A. Romanenko, T. Raubenheimer and, V. Yakovlev, "Wakefields in the Superconducting RF Cavities of LCLS-II,", in proceedings of LINAC2014, Geneva, Switzerland, THPP124, pp. 1147-50.

[14] A. Chao and, M. Tigner, "Handbook of Accelerator Physics and Engineering," World Scientific, 1998, pp. 190-250.

[15] K.L.F. Bane, C. Nantista and C. Adolphsen, "Higher Order Mode Heating Analysis for the ILC Superconducting Linacs," in proceedings of LINAC2010, Tsukuba, Japan, THP021, pp. 803-05.

[16] "R. Brinkmann, M. Dohlus, D. Trines, A. Novokhatsky, M. Timm, T. Weiland, P. Hulsmann, C. T. Rieck, K. Scharnberg, P. Schmueser," Terahertz wakefields in the superconducting cavities of the TESLA-FEL linac," DESY, Germany, Rep. 2000-07, March, 2000.

[17] T. Weiland and I. Zagorodnov, "The Short-Range Transverse Wake Function For TESLA Accelerating Structure,” DESY, Germany, Rep. 2003-19, 2003.

[18] K. Bane, C. Adolphsen, C. Nantista, T. Raubenheimer, A. Saini, N. Solyak and, V. Yakovlev, "Distribution from Untrapped HOM radiation in the LCLSII cryomodules," Physics Procedia, vol. 79, pp. 13-20, 2015, DOI: https://doi.org/10.1016/j.phpro.2015.11.057.

[19] K. Bane, C. Adolphsen, C. Nantista, T. Raubenheimer, A. Saini, N. Solyak and, V. Yakovlev, "Untrapped HOM Radiation Absorption in the LCLS-II Cryomodules," in proceedings of LINAC2014, Geneva, Switzerland, MOPP126, pp. 351-54.

[20] A. Saini, A. Lunin, N. Solyak, A. Sukhanov and, V. Yakovlev, "RF Losses in $1.3 \mathrm{GHz}$ Cryomodules of the LCLS-II Superconducting CW Linac," in proceedings of LINAC2016, Lansing, MI, USA, THPRC014, pp. 798-801.

[21] ANSYS, Inc., http://www.ansys.com/Products/Electronics/ ANSYS-HFSS, 2017

[22] https://trc.nist.gov/cryogenics/materials/OFHC\%20Copper/OFHC Coppe r rev1.htm 